\title{
A Molecular Genetic Map and Electrophoretic Karyotype of the Plant Pathogenic Fungus Cochliobolus sativus
}

\author{
Shaobin Zhong, ${ }^{1}$ Brian J. Steffenson, ${ }^{1}$ J. Patrick Martinez, ${ }^{2}$ and Lynda M. Ciuffetti ${ }^{2}$ \\ ${ }^{1}$ Department of Plant Pathology, North Dakota State University, Fargo 58105; and ${ }^{2}$ Department of Botany and Plant \\ Pathology, Oregon State University, Corvallis 97331
}

Submitted 12 July 2001. Accepted 14 January 2002.

\begin{abstract}
A molecular genetic map was constructed and an electrophoretic karyotype was resolved for Cochliobolus sativus, the causal agent of spot blotch of barley and wheat. The genetic map consists of 27 linkage groups with 97 amplified fragment length polymorphism (AFLP) markers, 31 restriction fragment length polymorphism (RFLP) markers, two polymerase chain reaction amplified markers, the mating type locus (CsMAT), and a gene ( $V H v 1)$ conditioning high virulence on barley cv. Bowman. These linkage groups covered a map distance of $849 \mathrm{cM}$. The virulence gene $V H v 1$ cosegregated with six AFLP markers and was mapped on one of the major linkage groups. Fifteen chromosome-sized DNAs were resolved in $C$. sativus isolates ND93-1 and ND90Pr with contour-clamped homogeneous electric field (CHEF) electrophoresis combined with telomere probe analysis of comigrating chromosome-sized DNAs. The chromosome sizes ranged from 1.25 to 3.80 Mbp, and the genome size of the fungus was estimated to be approximately $33 \mathrm{Mbp}$. By hybridizing genetically mapped RFLP and AFLP markers to CHEF blots, 25 of the 27 linkage groups were assigned to specific chromosomes. The barley-specific virulence locus $V H v 1$ was localized on a chromosome of $2.80 \mathrm{Mbp}$ from isolate ND90Pr in the CHEF gel. The total map length of the fungus was estimated to be at least $1,329 \mathrm{cM}$ based on the map distance covered by the linked markers and the estimated gaps. Therefore, the physical to genetic distance ratio is approximately $25 \mathrm{~kb} / \mathrm{cM}$. Construction of a high-resolution map around target loci will facilitate the cloning of the genes conferring virulence and other characters in $C$. sativus by a map-based cloning strategy.
\end{abstract}

Genetic linkage maps not only provide a fundamental basis for understanding genome organization and evolution, but also are essential for cloning and characterizing genes of interest based on a map-based cloning strategy (Young 1990). Several types of molecular markers have proven useful in genetic map construction. These include restriction fragment length polymorphism (RFLP) markers (Botstein et al. 1980) and polymerase chain reaction (PCR)-based markers, such as randomly amplified polymorphic DNAs (RAPDs) (Williams et al. 1991) and amplified fragment length polymorphisms (AFLPs) (Vos et al. 1995). With these different markers, molecular genetic maps have been constructed for a number of plant pathogenic

Corresponding author: Brian J. Steffenson, E-mail: bsteffen@umn.edu

Current address of Shaobin Zhong and Brian J. Steffenson: Department of Plant Pathology, University of Minnesota, St. Paul 55108. fungi, such as the lettuce downy mildew pathogen Bremia lactucae (Hulbert et al. 1988), the southern corn leaf blight pathogen Cochliobolus heterostrophus (Tzeng et al. 1992), the rice blast pathogen Magnaporthe grisea (Skinner et al. 1993), the soybean root rot pathogen Phytophthora sojae (Whisson et al. 1995), the maize seedling blight and root rot pathogen Gibberella fujikuroi (mating population A) (Xu and Leslie 1996), the potato late blight pathogen Phytophthora infestans (van der Lee et al. 1997), and the crucifer black leg pathogen Leptosphaeria maculans (Pongam et al. 1999). Development of genetic maps and the identification of molecular markers closely linked to target genes have allowed for the map-based cloning of several genes conferring specificity to different grass species and rice cultivars in $M$. grisea (Farman and Leong 1998; Orbach et al. 2000; Sweigard et al. 1995; Valent 1997).

Cochliobolus sativus (Ito \& Kuribayashi) Drechs. ex Dastur (anamorph: Bipolaris sorokiniana (Sacc.) Shoemaker) is a fungal pathogen that causes several important diseases (spot blotch, kernel blight, and common root rot) of barley and wheat (Mathre 1997; Wiese 1977). Due to the economic importance of the diseases caused by this pathogen, extensive studies have been conducted on the biology of $C$. sativus at the genetic and molecular level. These include genetic analysis of virulence and pathogenicity (Hosford et al. 1975; Kline and Nelson 1971; Valjavec-Gratian and Steffenson 1997), cloning of mating type genes (Zhong and Steffenson 2001a), and characterization of virulence and DNA diversity (Zhong and Steffenson 2001b). The objectives of this study were to construct a molecular genetic map of $C$. sativus using AFLP and RFLP markers and to resolve the chromosomes of the fungus by electrophoretic karyotype analysis.

\section{RESULTS}

AFLP markers.

Sixty-four primer combinations of eight EcoRI primers and eight MseI primers were tested on the parental isolates ND90Pr and ND93-1. Primer pairs (20) generating the greatest polymorphism were used on the progeny population, and a total of 115 segregating AFLP markers were identified. Of the AFLP markers, $101(82.6 \%)$ showed a 1:1 Mendelian segregation ratio in the progeny population (Table 1). In all, 14 markers $(17.4 \%)$ exhibited a distorted segregation ratio, 11 at the $5 \%$ significance level and 3 at the $1 \%$ significance level.

RFLP markers.

DNA clones from C. heterostrophus (Tzeng et al. 1992) were used as probes to hybridize with restriction enzyme digests of DNA from the parental C. sativus isolates ND90Pr 
and ND93-1. Of the 79 clones surveyed, 75 (95\%) showed strong hybridization with genomic DNA of C. sativus, and 44 $(56 \%)$ detected polymorphisms between the two parental isolates using at least one of the five restriction enzymes: BamHI, $E c o$ RI, EcoRV, PvuII, and XhoI. The frequency of RFLPs revealed by different enzymes varied, with EcoRV yielding the highest $(42.7 \%)$ and XhoI the lowest (26.7\%) number of RFLPs

All of the types of RFLPs detected by Tzeng and associates (1992) in $C$. heterostrophus also were observed in $C$. sativus. These included RFLPs identified by a repetitive sequence, a single copy sequence present in both parents, a single copy sequence present in one parent, and a single copy sequence located in different chromosomal positions in the two parents. Of the 44 clones that produced polymorphisms between the parents, 20 were used to assess polymorphisms in the progeny population, and 38 RFLP markers were identified. Of these RFLP markers, $32(84.2 \%)$ segregated in a $1: 1$ ratio. Six RFLP markers exhibited distorted segregation, two at the $5 \%$ significance level and four at the $1 \%$ significance level (Table 1).
Other markers.

Virulence loci. ND90Pr and ND93-1 exhibited high and low virulence, respectively, on barley cv. Bowman (ValjavecGratian and Steffenson 1997). Virulence assessments of the 104 progeny isolates indicated that a single locus (designated $V H v 1$ ) in isolate ND90Pr controls high virulence on 'Bowman' (Valjavec-Gratian and Steffenson 1997). From the evaluation of progeny on wheat line PI 254042, 58 isolates exhibited low virulence (rating of 1 to 3 ) and 46 isolates exhibited high virulence (rating of 4 to 5$)\left(\chi^{2}=1.39 ; P=0.24\right.$; df $=1$ for $1: 1$ ratio), indicating that a single locus (designated VTal) in isolate ND93-1 controls virulence on wheat (Table 1).

Mating type locus. Mating type determinations of the progeny by backcrossing to the parents were mostly unsuccessful due to the infertility of mated isolates (Zhong and Steffenson 2001a). Instead, the mating type of progeny was determined by DNA hybridization using MAT-1- and MAT-2-specific DNA fragments as probes. DNA of 51 progeny hybridized with the $M A T-1$-specific probe, whereas DNA of the remaining 53 isolates hybridized with the MAT-2-specific probe (Zhong and Steffenson 2001a). Segregation for mating type in the progeny

Table 1. Genetic markers used for map construction in Cochliobolus sativus

\begin{tabular}{|c|c|c|c|c|c|c|c|c|c|}
\hline Locus $^{a}$ & Seg. ${ }^{b}$ & $\chi^{2}$ & $P^{\mathrm{c}}$ & Chrom. $^{d}$ & Locus $^{\mathrm{a}}$ & Seg. ${ }^{b}$ & $\chi^{2}$ & $P^{\mathbf{c}}$ & Chrom. $^{d}$ \\
\hline Mating type locus & & & & & E-AA/M-CT-750 & $48: 55$ & 0.48 & 0.49 & 1 \\
\hline CsMAT (MAT-1/MAT-2) & $51: 53$ & 0.04 & 0.85 & 8 & E-AA/M-CT-505 & $43: 60$ & 2.81 & 0.10 & 1 \\
\hline Virulence locus ${ }^{\mathrm{f}}$ & & & & & E-AA/M-CT-397 & $46: 57$ & 1.18 & 0.28 & 11 \\
\hline$V H v 1$ & $49: 55$ & 0.35 & 0.56 & 8 & E-AA/M-CT-290 & $56: 47$ & 0.79 & 0.38 & 7 \\
\hline VTa1 & $58: 46$ & 1.39 & 0.24 & $\ldots$ & E-AA/M-CT-208 & $41: 62$ & 4.28 & $0.04 *$ & 8 \\
\hline PCR marker ${ }^{\mathrm{g}}$ & & & & & E-AA/M-CT-173 & $43: 60$ & 2.81 & 0.10 & 1 \\
\hline HMGPCR1 & $44: 59$ & 1.18 & 0.28 & 6 & E-AA/M-CT-128 & $43: 60$ & 2.81 & 0.10 & 1 \\
\hline HMGPCR2 & $44: 59$ & 2.18 & 0.14 & 6 & E-AG/M-CC-754 & $54: 50$ & 0.15 & 0.70 & 1 \\
\hline AFLP marker ${ }^{\mathrm{h}}$ & & & & & E-AG/M-CC-753 & $54: 50$ & 0.15 & 0.70 & 1 \\
\hline E-AG/M-CG-405 & $55: 48$ & 0.48 & 0.49 & $\ldots$ & E-AG/M-CC-402 & $48: 55$ & 0.48 & 0.50 & 8 \\
\hline E-AG/M-CG-293 & $59: 44$ & 2.18 & 0.14 & 2 & E-AT/M-CT-708 & $57: 47$ & 0.96 & 0.33 & 9 \\
\hline E-AG/M-CG-278 & $49: 54$ & 0.24 & 0.62 & 5 & E-AT/M-CT-353 & $48: 56$ & 0.62 & 0.43 & 8 \\
\hline E-AG/M-CG-121 & $48: 55$ & 0.48 & 0.49 & 8 & E-AT/M-CT-116 & $56: 48$ & 0.62 & 0.43 & $\ldots$ \\
\hline E-AG/M-CG-110 & $47: 54$ & 0.49 & 0.49 & $\ldots$ & E-AT/M-CT-110 & $42: 62$ & 3.85 & $0.05 *$ & $\ldots$ \\
\hline E-AA/M-CC-783 & $53: 50$ & 0.09 & 0.77 & 1 & E-AC/M-CT-708 & $52: 51$ & 0.01 & 0.92 & 10 \\
\hline E-AA/M-CC-614 & $52: 51$ & 0.01 & 0.92 & 8 & E-AC/M-CT-298 & $56: 48$ & 0.62 & 0.43 & 2 \\
\hline E-AA/M-CC-300 & $53: 51$ & 0.04 & 0.85 & 1 & E-AC/M-CT-193 & $48: 56$ & 0.62 & 0.43 & LG 26 \\
\hline E-AA/M-CC-242 & $45: 59$ & 1.89 & 0.17 & 1 & E-AC/M-CT-166 & $47: 57$ & 0.96 & 0.33 & $\ldots$ \\
\hline E-AA/M-CC-139 & $54: 50$ & 0.15 & 0.70 & 4 & E-AC/M-CT-165 & $52: 52$ & 0.00 & 1.00 & $\ldots$ \\
\hline E-AA/M-CC-138 & $42: 62$ & 3.85 & $0.05^{*}$ & 11 & E-AC/M-CT-158 & $54: 50$ & 0.15 & 0.69 & $\ldots$ \\
\hline E-AA/M-CC-137 & $42: 62$ & 3.85 & $0.05^{*}$ & 11 & E-AT/M-CA-686 & $45: 59$ & 1.89 & 0.17 & 11 \\
\hline E-AA/M-CC-136 & $65: 39$ & 6.50 & $0.01 *$ & 5 & E-AT/M-CA-678 & $54: 50$ & 0.15 & 0.69 & 12 \\
\hline E-AA/M-CC-135 & $42: 62$ & 3.85 & $0.05^{*}$ & 11 & E-AT/M-CA-317 & $48: 56$ & 0.62 & 0.43 & 8 \\
\hline E-AA/M-CG-809 & $47: 55$ & 0.63 & 0.43 & 1 & E-AT/M-CA-295 & $72: 31$ & 16.32 & $0.00 * *$ & LG 27 \\
\hline E-AT/M-CG-760 & $53: 51$ & 0.04 & 0.85 & $\ldots$ & E-AT/M-CA-290 & $55: 49$ & 0.35 & 0.56 & 1 \\
\hline E-AT/M-CG-475 & $47: 57$ & 0.96 & 0.33 & 11 & E-AC/M-CC-483 & $46: 58$ & 1.39 & 0.24 & 8 \\
\hline E-AT/M-CG-318 & $61: 43$ & 3.12 & 0.08 & 2 & E-AC/M-CC-472 & $46: 58$ & 1.39 & 0.24 & 8 \\
\hline E-AA/M-CT-1072 & $47: 56$ & 0.79 & 0.38 & LG 26 & E-AC/M-CC-275 & $56: 48$ & 0.62 & 0.43 & 6 \\
\hline E-AA/M-CT-1071 & $47: 56$ & 0.79 & 0.38 & LG 26 & E-AC/M-CC-188 & $61: 43$ & 3.12 & 0.08 & 2 \\
\hline E-AA/M-CT-751 & $48: 55$ & 0.48 & 0.49 & 1 & E-AC/M-CG-910 & $49: 55$ & 0.35 & 0.56 & 8 \\
\hline & & & & & & & & \multicolumn{2}{|c|}{ (continued on next page } \\
\hline
\end{tabular}

${ }^{a}$ One locus has two alleles (presence/absence or two alternate genotypes).

b Segregation for the mating type locus (CsMAT) is given in the form of MAT-1 versus MAT-2; for the virulence loci (VHv1 and VTa1), segregation data are given as high virulence versus low virulence on each of the two differential hosts (barley cv. Bowman and wheat PI 254042); segregation data for all DNA markers are in the form of presence versus absence.

${ }^{c}$ Significant deviation from a 1:1 Mendelian segregation ratio was tested by the chi-square test; $*$ and $* *$ indicate significant deviations at the 5 and $1 \%$ level, respectively.

${ }^{\mathrm{d}}$ Chromosomes were resolved with contour-clamped homogeneous electric field (CHEF) and telomere probe hybridization. Chromosomes in isolates ND93-1 were used as a reference and are numbered in order of decreasing size. Location of markers was determined by hybridization to the CHEF-separated chromosomes; LG = linkage group; ... indicates data not available or applicable.

e Mating type was determined by DNA hybridization with MAT-1 and MAT-2 specific probes (Zhong and Steffenson 2001a).

${ }^{\mathrm{f}} \mathrm{VHv} 1$ and VTal are loci conferring high virulence on barley cv. Bowman and wheat line PI 254042, respectively.

g Polymerase chain reaction (PCR) markers were amplified with primer pair CsPrimer1 and CsPrimer2 (Zhong and Steffenson 2001a).

${ }^{\mathrm{h}}$ Amplified fragment length polymorphism (AFLP) marker exhibits presence or absence of alleles.

${ }^{\mathrm{i}}$ Restriction fragment length polymorphism (RFLP) markers were detected using DNA clones from C. heterostrophus (Tzeng et al. 1992) and were scored as presence or absence of alleles. When an RFLP marker showed co-dominance (two alternative bands), only one band was scored. Each RFLP marker was named based on the clone designation followed by the restriction enzyme $(\mathrm{B}, \mathrm{EI}, \mathrm{EV}, \mathrm{P}$, and $\mathrm{X}) \mathrm{used} . \mathrm{B}=B a m \mathrm{HI}, \mathrm{EI}=E c o \mathrm{RI}, \mathrm{EV}=E c o \mathrm{RV}, \mathrm{P}=P v u \mathrm{II}$, and $\mathrm{X}=$ XhoI. The suffix ( $\mathrm{a}, \mathrm{b}, \mathrm{c}$, and so on) indicates different RFLP markers detected by the same probe-enzyme combination. 
population fit a 1:1 ratio (Table 1), indicating the involvement of a single gene. The mating type locus was designated as CsMAT.

PCR-amplified markers. The primer pairs CsPrimer1 and CsPrimer2 (Zhong and Steffenson 2001a), used to amplify the Cochliobolus HMG box (Arie et al. 1997; Turgeon et al. 1993), also generated two other PCR products (HMGPCR1 and HMGPCR2) that were polymorphic between parental isolates ND90Pr and ND93-1. HMGPCR1 only was present in ND93-1, and HMGPCR2 only was present in ND90Pr. PCR amplification with DNA from the progeny population showed a 1:1 segregation ratio for the presence of and absence of these DNA fragments (Table 1).

\section{Linkage analysis and map construction.}

A total of 158 polymorphic markers were used in the linkage analysis to construct a genetic map of $C$. sativus. These included 115 AFLP markers, 38 RFLP markers, 2 PCR-amplified DNA markers (HMGPCR1 and HMGPCR2), the matingtype locus (CsMAT), and two virulence loci (VHvl and VTal). When a minimum logarithm of odds (LOD) value of 4.0 and a maximum theta of 0.3 were used in the MAPMAKER program, 134 markers were joined into 27 linkage groups with at least two markers in each linkage group (Fig. 1). Twenty-four markers remained unlinked (Table 1), including the virulence locus VTal for wheat, and were not included in the genetic map. The linked markers covered a total genetic distance of $849.4 \mathrm{cM}$. Linkage groups ranged from 0 to $89.2 \mathrm{cM}$ in size. The locus conferring virulence on barley $(V H v 1)$ was mapped to one of the linkage groups (linkage group 17) and cosegregated with six AFLP markers (Fig. 1).

\section{Electrophoretic karyotype analysis.}

Electrophoretic karyotype analysis was performed on isolates ND90Pr, ND93-1, and five progeny isolates $\left(\mathrm{F}_{1}-1, \mathrm{~F}_{1}-2\right.$, $F_{1}-5, F_{1}-61$, and $\left.F_{1}-62\right)$ from the ND90Pr $\times$ ND93-1 cross. Progeny $F_{1}-1, F_{1}-2$, and $F_{1}-61$ exhibited high virulence, whereas $F_{1}-5$ and $F_{1}-62$ exhibited low virulence on barley cv. Bowman. Under the same electrophoretic conditions, the banding patterns were highly reproducible for the same isolates but differed dramatically among isolates. Eight chromosome-sized bands were observed with isolate ND93-1, and

Table 1. (continued from preceding page)

\begin{tabular}{|c|c|c|c|c|c|c|c|c|c|}
\hline Locus $^{a}$ & Seg. & $\chi^{2}$ & $P^{\mathrm{c}}$ & Chrom. $^{d}$ & Locus $^{a}$ & Seg. ${ }^{b}$ & $\chi^{2}$ & $P^{\mathrm{c}}$ & Chrom. $^{d}$ \\
\hline E-AC/M-CG-343 & $51: 53$ & 0.04 & 0.85 & $\ldots$ & E-GG/M-AC-376 & $50: 53$ & 0.09 & 0.77 & 8 \\
\hline E-AC/M-CG-335 & $48: 56$ & 0.62 & 0.43 & 11 & E-GG/M-AC-286 & $50: 53$ & 0.09 & 0.77 & 10 \\
\hline E-AC/M-CG-195 & $45: 59$ & 1.89 & 0.17 & 1 & E-GG/M-AC-284 & $48: 55$ & 0.48 & 0.49 & 10 \\
\hline E-AT/M-CC-611 & $43: 60$ & 2.81 & 0.09 & 8 & E-GT/M-AG-366 & $57: 46$ & 1.18 & 0.28 & $\ldots$ \\
\hline E-AT/M-CC-501 & $50: 54$ & 0.15 & 0.69 & 10 & E-GT/M-AG-247 & $71: 32$ & 14.77 & $0.00 * *$ & LG 27 \\
\hline E-AT/M-CC-499 & $50: 54$ & 0.15 & 0.69 & $\ldots$ & E-GT/M-AG-168 & $47: 56$ & 0.79 & 0.38 & 11 \\
\hline E-AT/M-CC-182 & $45: 59$ & 1.89 & 0.17 & 13 & E-GT/M-AG-109 & $46: 57$ & 1.18 & 0.28 & 8 \\
\hline E-AA/M-CA-468 & $42: 62$ & 3.85 & $0.05^{*}$ & 11 & E-GT/M-AG-105 & $44: 59$ & 2.18 & 0.14 & 8 \\
\hline E-AA/M-CA-391 & $47: 57$ & 0.96 & 0.33 & 1 & RFLP marker $^{\mathrm{i}}$ & & & & \\
\hline E-AA/M-CA-141 & $58: 46$ & 1.39 & 0.24 & $\ldots$ & B84EV & $62: 42$ & 3.85 & $0.05 *$ & 2 \\
\hline E-AA/M-CA-133 & $48: 56$ & 0.62 & 0.43 & $\ldots$ & B88EV-a & $41: 60$ & 3.57 & 0.06 & 13 \\
\hline E-AG/M-CA-207 & $49: 55$ & 0.35 & 0.56 & 8 & B88EV-b & $42: 55$ & 1.74 & 0.19 & 9 \\
\hline E-AG/M-CA-200 & $47: 57$ & 0.96 & 0.33 & 5 & B88EV-c & $42: 59$ & 2.86 & 0.09 & 13 \\
\hline E-AG/M-CA-196 & $47: 57$ & 0.96 & 0.33 & 5 & B88EV-d & $44: 58$ & 1.92 & 0.17 & 4 \\
\hline E-AG/M-CA-171 & $48: 48$ & 0.00 & 1.00 & 12 & B88EV-e & $52: 52$ & 0.00 & 1.00 & 6 \\
\hline E-AG/M-CT-703 & $49: 54$ & 0.24 & 0.62 & 8 & B88EV-f & $55: 47$ & 0.63 & 0.43 & $\ldots$ \\
\hline E-AG/M-CT-443 & $41: 63$ & 4.65 & $0.03 *$ & 8 & B 107P & $53: 41$ & 1.53 & 0.22 & 2 \\
\hline E-AG/M-CT-382 & $61: 43$ & 3.12 & 0.08 & 2 & B114EV & $42: 58$ & 2.56 & 0.11 & 11 \\
\hline E-AG/M-CT-97 & $48: 56$ & 0.62 & 0.43 & 10 & B149EV-a & $56: 45$ & 1.20 & 0.27 & $\ldots$ \\
\hline E-AC/M-AT-882 & $56: 46$ & 0.98 & 0.32 & 10 & B149EV-b & $58: 41$ & 2.92 & 0.09 & 2 \\
\hline E-AC/M-AT-577 & $53: 50$ & 0.09 & 0.77 & $\ldots$ & B149EV-c & $47: 54$ & 0.46 & 0.49 & $\ldots$ \\
\hline E-AC/M-AT-403 & $43: 60$ & 2.81 & 0.09 & LG 27 & B421B & $59: 44$ & 2.18 & 0.14 & 3 \\
\hline E-AC/M-AT-305 & $49: 53$ & 0.16 & 0.69 & 8 & B403EI & $36: 46$ & 1.22 & 0.27 & 4 \\
\hline E-AC/M-AT-304 & $49: 53$ & 0.16 & 0.69 & 8 & B405EV & $55: 49$ & 0.35 & 0.56 & 4 \\
\hline E-AC/M-AT-278 & $49: 54$ & 0.24 & 0.62 & 5 & C9EI-a & $41: 54$ & 1.78 & 0.18 & 3 \\
\hline E-AC/M-AT-263 & $44: 59$ & 2.18 & 0.14 & $\ldots$ & C9EI-b & $52: 43$ & 0.85 & 0.36 & 3 \\
\hline E-AC/M-AT-261 & $50: 53$ & 0.09 & 0.77 & $\ldots$ & C70EV & $59: 45$ & 1.89 & 0.17 & 2 \\
\hline E-AT/M-AC-1062 & $60: 38$ & 4.94 & $0.03 *$ & $\ldots$ & C193B & $38: 66$ & 7.54 & $0.01 * *$ & 12 \\
\hline E-AT/M-AC-638 & $54: 46$ & 0.64 & 0.42 & 2 & G29P & $42: 43$ & 0.01 & 0.91 & 7 \\
\hline E-AT/M-AC-637 & $54: 46$ & 0.64 & 0.42 & 2 & G98EV-a & $52: 49$ & 0.09 & 0.77 & 2 \\
\hline E-AT/M-AC-584 & $55: 46$ & 0.80 & 0.37 & 1 & G98EV-b & $43: 61$ & 3.12 & 0.08 & 11 \\
\hline E-AT/M-AC-583 & $55: 46$ & 0.80 & 0.37 & 1 & G98EV-c & $43: 61$ & 3.12 & 0.08 & 11 \\
\hline E-AT/M-AC-271 & $39: 62$ & 5.24 & $0.02 *$ & 9 & G98EV-d & $43: 61$ & 3.12 & 0.08 & 11 \\
\hline E-AT/M-AC-211 & $47: 49$ & 0.04 & 0.84 & 6 & G127X & $38: 41$ & 0.11 & 0.74 & 4 \\
\hline E-AT/M-AC-191 & $40: 60$ & 4.00 & $0.05^{*}$ & 8 & G131EI-a & $62: 33$ & 8.85 & $0.00 * *$ & 1 \\
\hline E-AT/M-AC-158 & $44: 57$ & 1.67 & 0.20 & 13 & G131EI-b & $46: 49$ & 0.10 & 0.76 & 1 \\
\hline E-GC/M-AG-633 & $44: 59$ & 2.18 & 0.14 & 11 & G213EV-a & $55: 49$ & 0.35 & 0.56 & 3 \\
\hline E-GC/M-AG-508 & $55: 48$ & 0.48 & 0.49 & $\ldots$ & G213EV-b & $51: 50$ & 0.01 & 0.92 & $\ldots$ \\
\hline E-GC/M-AG-237 & $53: 50$ & 0.09 & 0.77 & 3 & G213EV-c & $43: 58$ & 2.23 & 0.14 & LG 27 \\
\hline E-GC/M-AG-172 & $51: 52$ & 0.01 & 0.92 & 12 & G213EV-d & $47: 57$ & 0.96 & 0.33 & 11 \\
\hline E-GC/M-AG-170 & $51: 52$ & 0.01 & 0.92 & 12 & G309P-a & $41: 45$ & 0.19 & 0.67 & 6 \\
\hline E-GC/M-AG-115 & $46: 57$ & 1.18 & 0.28 & 8 & G309P-b & $55: 31$ & 6.70 & $0.01 * *$ & 11 \\
\hline E-GC/M-AG-104 & $49: 54$ & 0.24 & 0.62 & 8 & G311B-a & $61: 42$ & 3.51 & 0.06 & 3 \\
\hline E-GC/M-AG-82 & $49: 54$ & 0.24 & 0.62 & 8 & G311B-b & $59: 44$ & 2.18 & 0.14 & 3 \\
\hline E-GG/M-AC-925 & $50: 22$ & 0.04 & 0.84 & 3 & G353EI-a & $28: 52$ & 7.20 & $0.01 * *$ & 4 \\
\hline E-GG/M-AC-813 & $50: 52$ & 0.04 & 0.84 & 3 & G353EI-b & $38: 42$ & 0.20 & 0.65 & 4 \\
\hline E-GG/M-AC-496 & $58: 44$ & 1.92 & 0.17 & 3 & G353EI-c & $30: 50$ & 5.00 & $0.03 *$ & 11 \\
\hline E-GG/M-AC-495 & $58: 44$ & 1.92 & 0.17 & 3 & & & & & \\
\hline
\end{tabular}


Chromosome 1
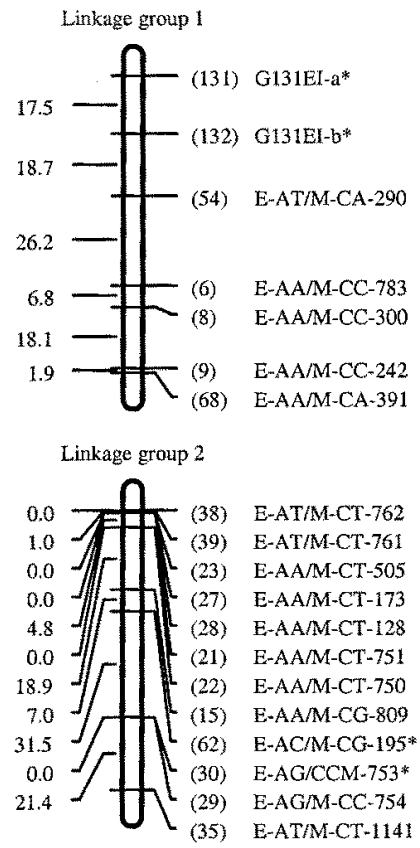

\footnotetext{
Linkage group 3

$0.0-\mathrm{OT}_{(96)} \begin{aligned} & \text { E-ATM-AC-584* } \\ & \text { E-AT/M+AC-583 }\end{aligned}$

B264

$\mathrm{C} 50$
}

Chromosome 5

$\begin{aligned} & \text { Linkage group } 11 \\ & 0.0 \\ & 17.0 \\ & 0.0\end{aligned}- \begin{cases}(89) & \text { E-ACM-AT-278 } \\ (3) & \text { E-AGM-CG-278 } \\ (72) & \text { E-AG/M-CA-200 } \\ (73) & \text { E-AGM-CA-196 }\end{cases}$

Linkage group 12

$1.0-\mathrm{O}_{(13)} \begin{array}{ll}\text { E-AG/M-CC-1 } 148^{*} & \text { E-AA/M-CC-136 }\end{array}$

B154

B301
Chromosome 2
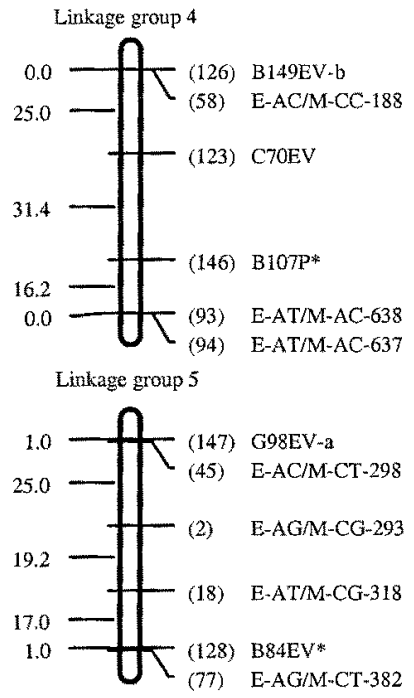

Chromosome 3

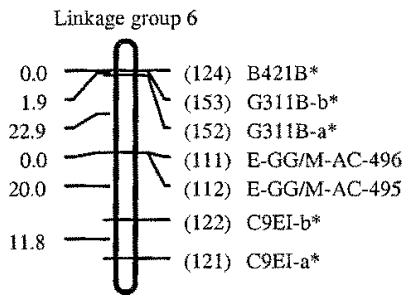

Linkage group 7

$30.5-\int_{(140) \text { G213EV-a }}^{(103) \text { E-GC/M-AG-237* }}$

Linkage group 8

$0.0-\mathrm{OT}_{(109)}$ E-GG/M-AC-925
Chromosome 4

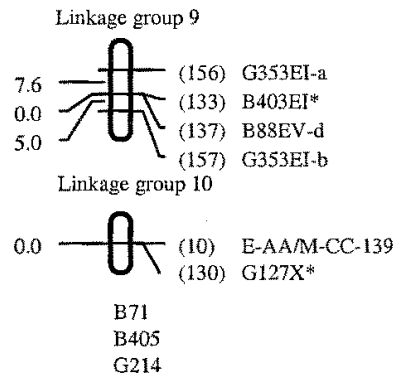

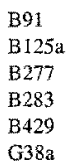

Chromosome 7

Linkage group 14

B246

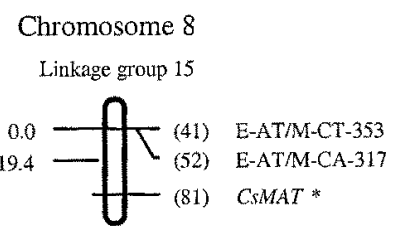

Linkage group 16

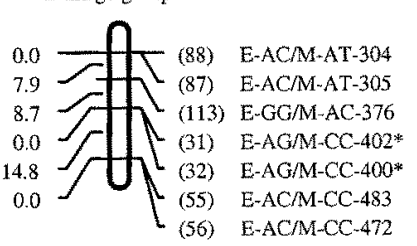

$\left.\begin{array}{rl}4.9 \\ 0.0 \\ 0.0 \\ 0.0 \\ 0.0 \\ 0.0 \\ 0.0 \\ 25.0 \\ 1.9 \\ 1.0 \\ 0.0 \\ 1.0 \\ 28.3 \\ 17.4 \\ 0.0\end{array}\right]=\left[\begin{array}{ll}(7) & \text { E-AA/M-CC-614 } \\ (4) & \text { E-AG/M-CG-121* } \\ (59) & \text { E-AC/M-CG-910 } \\ (71) & \text { E-AG/M-CA-207* } \\ (82) & \text { VHul } \\ (107) & \text { E-GC/M-AG-104 } \\ (108) & \text { E-GC/M-AG-82 } \\ (75) & \text { E-AG/M-CT-703 } \\ (120) & \text { E-GT/M-AG-105 } \\ (26) & \text { E-AA/M-CT-208 } \\ (99) & \text { E-AT/M-AC-191 } \\ (76) & \text { E-AG/M-CT-443 } \\ (63) & \text { E-AT/M-CC-611 } \\ (106) & \text { E-GC/M-AG-115 } \\ (119) & \text { E-GT/M-AG-109 }\end{array}\right.$

Fig. 1. Genetic map of Cochliobolus sativus based on 97 amplified fragment length polymorphism (AFLP) markers, 31 restriction fragment length polymorphism (RFLP) markers, two polymerase chain reaction-amplified markers, the mating type locus (CsMAT), and the barley cultivar-specific virulence locus ( VHv1). Twenty-seven linkage groups (1 to 27) are included. Of the 27 linkage groups, 25 (excluding groups 26 and 27 ) were assigned to 13 of the 15 chromosomes based on hybridization of mapped markers (indicated with an asterisk) with electrophoretically separated chromosomes. Two linkage groups (26 and 27) were not assigned to specific chromosomes because the AFLP markers cloned from these groups were repeated sequences and showed hybridization to all chromosomes. The orientation of the linkage groups assigned to the same chromosome is not known. C. heterostrophus clones that were not mapped genetically, but hybridized to electrophoretically separated chromosomes, were placed under the associated chromosomes. 
nine chromosome-sized bands were observed with isolate ND90Pr under contour-clamped homogeneous electric field (CHEF) conditions for resolving higher molecular weight bands (2.5 to $4.0 \mathrm{Mbp}$ ) (Fig. 2). The two most rapidly migrating bands of ND90Pr were further resolved into four bands using $\mathrm{CHEF}$ conditions for resolving lower molecular weight bands (1.0 to $2.5 \mathrm{Mbp}$ ) (data not shown). Combining the results from both CHEF conditions, 8 chromosome bands were resolved for isolate ND93-1, and 11 chromosome bands were resolved for isolate ND90Pr. Chromosome bands were designated as A to $\mathrm{H}$ for isolate ND93-1 and as A to K for isolate ND90Pr, based on their descending magnitude of size (Fig. 2).

Telomere analysis of CHEF-separated chromosome bands.

Some CHEF-separated chromosome bands showed a higher staining intensity than other chromosome bands (e.g., band F in isolate ND93-1 and bands D and G in isolate ND90Pr), suggesting that they might contain more than one chromosome (Fig. 2). To more clearly resolve the number of chromosomes in each CHEF-separated band, DNA was isolated from each band, digested with BamHI, separated in an agarose gel, blotted, and probed with the telomere probe pNla-17. Each chromosome arm contains a telomere; therefore, two telomere hybridization bands were expected for each chromosome. In isolate ND93-1, chromosome bands A, B, and C contained one chromosome because each showed two telomere hybridization bands (Fig. 3); chromosome bands D, G, and H contained two chromosomes because each showed two telomere bands plus one high-intensity band which may suggest a doublet (Fig. 3); chromosome band E contained two chromosomes because it showed four telomere hybridization bands; and chromosome band $\mathrm{F}$ contained four chromosomes because it showed six telomere bands, two of which had higher intensity suggesting a doublet. In isolate ND90Pr, all chromosome bands contained only one chromosome, except bands D and G containing two and four chromosomes, respectively (data not shown). Based on the number of DNA fragments that hybridized with the telomere probe, 15 chromosomes were resolved in both isolates ND93-1 and ND90Pr (Table 2). The chromosome number also was resolved by the telomere probe with another restriction enzyme HindIII (data not shown) and confirmed by hybridization of RFLP and AFLP markers to CHEF blots of isolates ND93-1 and ND90Pr (discussed below). Using chromosomes of Schizosaccharomyces pombe and Hansenula wingei as standards, the chromosome sizes of $C$. sativus ranged from 1.25 to $3.8 \mathrm{Mbp}$. The estimated genome size was 33.7 Mbp in isolate ND90Pr and 33.4 Mbp in isolate ND93-1.

\section{Association of linkage groups and DNA markers with CHEF-separated chromosomes.}

To associate the linkage groups based on DNA markers (Fig. 1) with specific chromosomes resolved by CHEF (Fig. 2), at

Fig. 1. (continued from preceding page)

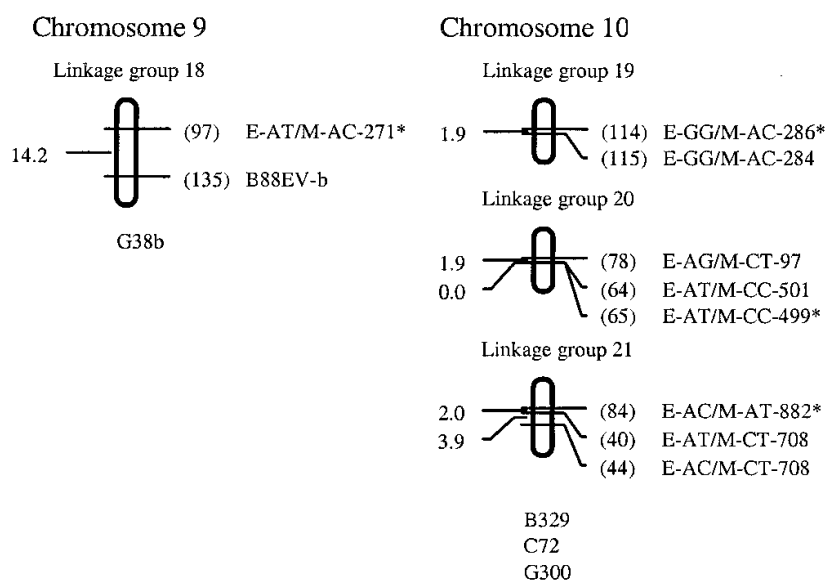

Chromosome 14

B257

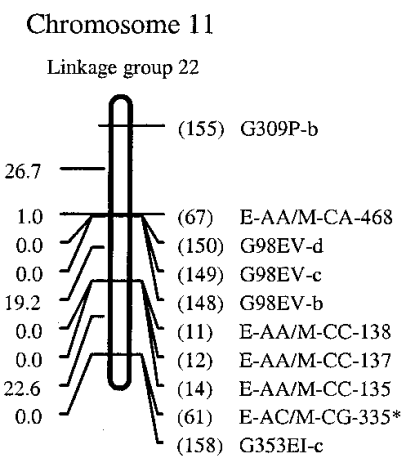

Chromosome 12

$$
\text { Linkage group } 24
$$

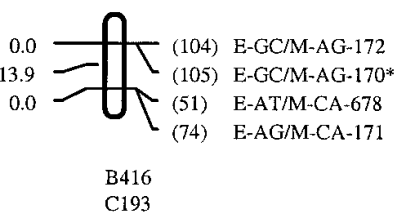

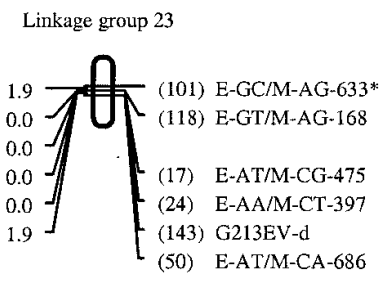

B43
B114

$$
\text { Chromosome } 15
$$

G235

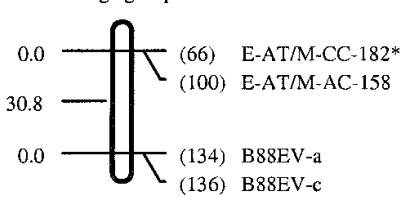

G199

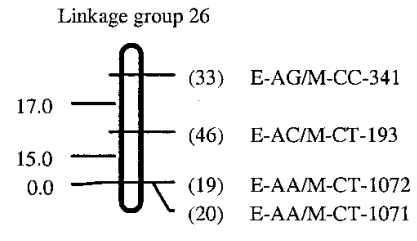

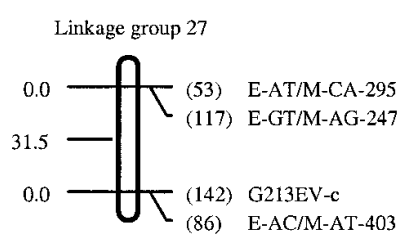


least one AFLP or RFLP marker from each linkage group was used as a probe to hybridize with the CHEF blots. Twentyeight $C$. heterostrophus clones, which hybridized with the $C$. sativus genome as a single copy but did not detect RFLPs between the two parental isolates, also were used to probe the CHEF blots to determine the homologous chromosomes between isolates ND93-1 and ND90Pr. Hybridization results are summarized in Table 2 . The hybridization patterns of representative probes on CHEF blots of the two parental isolates are shown in Figure 4A and B. Comparison of the hybridization patterns of the AFLP and RFLP clones on CHEF blots from isolates ND93-1 and ND90Pr indicated that some chromosomes that comigrated as one band in one isolate were separated in the other isolate. For example, chromosomes 4 and 5 of isolate ND93-1 comigrated as one chromosome band (D) but were separated into chromosome bands $\mathrm{D}$ and $\mathrm{H}$ in isolate ND90Pr, as revealed by hybridization with probes B71 and B154 (Table 2), respectively. When several chromosomes comigrated as one broad band, like band G in isolate ND90Pr, comparisons of hybridization patterns were made on the same CHEF blots to determine the identity of the chromosomes involved. This was done because probes representing different chromosomes in one isolate hybridized to different portions of the broad band in the other isolate. Using this approach, all 15 homologous chromosomes from the two parental isolates were identified (Fig. 5). Of the 27 linkage groups, 25 were assigned

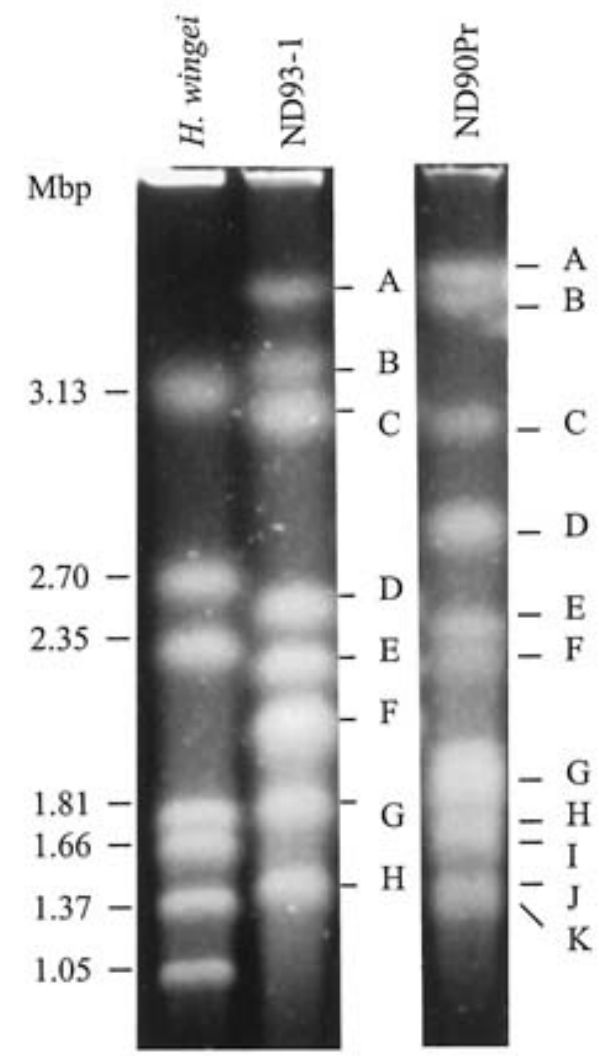

Fig. 2. Electrophoretically separated chromosomal DNAs of the Cochliobolus sativus parental isolates ND93-1 and ND90Pr under contour-clamped homogeneous electric field (CHEF) conditions for resolving higher molecular weight chromosome bands (2.5 to $4.0 \mathrm{Mbp}$ ). The two most rapidly migrating bands of isolate ND90Pr were further resolved into four bands using CHEF conditions for resolving lower molecular weight chromosome bands (1.0 to $2.5 \mathrm{Mbp}$ ) (data not shown). Chromosome bands are designated as A to $\mathrm{H}$ for isolate ND93-1 and as A to $\mathrm{K}$ for isolate ND90Pr based on their descending order of size. The chromosomes of Hansenula wingei were used as size markers and are indicated on the left. to 13 chromosomes, and all chromosomes were associated with at least one AFLP or RFLP marker (Figs. 1 and 5). Two linkage groups (26 and 27) were not associated with specific chromosomes because the AFLP markers cloned from these linkage groups were repeated sequences and showed hybridization to all the chromosomes (data not shown).

Some probes representing two different chromosomes in one isolate hybridized with one chromosome in the other isolate, suggesting that chromosome translocations occurred in the genome. For example, probes B107 and B285, which were localized on two separate chromosomes (2 and 6) of isolate ND93-1, hybridized with one chromosome (designated as 2;6) of isolate ND90Pr (Fig. 4A). Also, clones G311 and B405, which hybridized with chromosomes 3 and 4, respectively, of isolate ND93-1, were localized on the same chromosome (designated as 3;4) of isolate ND90Pr (Fig. 4A and B). Using the chromosomes of ND93-1 as a reference, five of the chromosomes in ND90Pr were found to be involved in translocations (Fig. 5).

The cloned AFLP marker E-AG/M-CA-207, which cosegregated with the virulence locus $V H v 1$, and the mating-type gene (CsMAT-1) were localized on the same chromosome (chromosome 8) of isolate ND90Pr (Fig. 6B and C, respectively). The estimated size of this chromosome was $2.80 \mathrm{Mbp}$. In isolate ND93-1, CsMAT-1 was localized on a chromosome of approximately $2.2 \mathrm{Mbp}$ (Fig. 6c). Thus, there was an approximately $600-\mathrm{kb}$ difference between these two homologous chromosomes.

\section{DISCUSSION}

Combining AFLP markers with RFLP markers, a genetic map of $C$. sativus was constructed which consists of 27 linkage groups that cover a total genetic map distance of $849.4 \mathrm{cM}$ (Fig. 1). The electrophoretic karyotype and telomere analyses revealed 15 chromosomes in isolates ND93-1 and ND90Pr; therefore, at least 15 gaps exist in the present genetic map of C. sativus. The maximum map length between two markers in the linkage groups was $31.5 \mathrm{cM}$; thus, each gap covers a map length of at least $32 \mathrm{cM}$. Based on this estimation, the total map length of $C$. sativus is at least $1,329 \mathrm{cM}$. The current genetic map, therefore, covers approximately $64 \%$ of the total map distance. The genome size of isolate ND93-1 is estimated to be $33.4 \mathrm{Mbp}$ based on comparisons with the standard chromosomes of $H$. wingei and $S$. pombe. Using $1,329 \mathrm{cM}$ as the total map length, the ratio of physical distance to genetic distance is estimated to be approximately $25 \mathrm{~kb} / \mathrm{cM}$. This value is in the range of 10 to $59 \mathrm{~kb} / \mathrm{cM}$ that was previously reported for other fungi such as Saccharomyces cereviseae $(10 \mathrm{~kb} / \mathrm{cM})$ (Fincham 1983), C. heterostrophus (23 kb/cM) (Tzeng et al. 1992), Bremia lactucae (25 kb/cM) (Hulbert et al. 1988), Fusarium monoliforme (32 kb/cM) (Xu and Leslie 1996), Neurospora crassa (43 kb/cM) (Orbach et al. 1988), and Magnaporthe grisea (40 to $45 \mathrm{~kb} / \mathrm{cM}$ ) (Skinner et al. 1993). However, the ratio of physical distance to genetic distance may vary with different chromosomes or chromosomal regions in a given species (Farman and Leong 1998; Xu and Leslie 1996).

Among the DNA clones from C. heterostrophus, 95\% hybridized with the genomic DNA of $C$. sativus, suggesting that high DNA similarity exists between the genomes of the two species. Some RFLP markers mapped on one chromosome of C. heterostrophus (Tzeng et al. 1992) were also localized on the same chromosome of C. sativus in a similar order (Fig. 1 and data not shown), indicating that synteny exists between chromosomes of the two species. Only 20 of the 44 C. heterostrophus clones that detected RFLPs between the two $C$. sativus parents were used and the RFLP markers detected were only 
mapped on some of the $C$. sativus linkage groups; therefore, a genome-wide synteny analysis between the two related species was not attempted. Use of additional RFLP markers that are distributed over all linkage groups of the two species would allow for comparative genome mapping and would provide useful information regarding the differentiation and evolution of genomes in Cochliobolus species. This approach has been widely used in plants to compare the genomes of cereal crops (Ahn and Tanksley 1993; Bennetzen and Freeling 1993; Devos et al. 1998) and those of tomato, potato, and pepper (Tanksley et al. 1992).

Previous cytological observations indicated that the haploid chromosome number of $C$. sativus was seven to eight (Hrushovetz 1956; Huang and Tinline 1974). However, our electrophoretic karyotype analysis combined with telomere hybridization strongly suggests that there are 15 chromosomes in this fungus. Discrepancies between cytological counts and electrophoretic analyses have been reported in several other fungi. For example, in C. heterostrophus, a haploid chromosome number of eight was reported by Guzman and associates (1982), who examined stained pachytene chromosomes under a light microscope; however, electrophoretic karyotyping, combined with molecular linkage analysis, resolved 15 to 16 chromosomes in this fungus (Tzeng et al. 1992). In Fusarium moniliforme, no more than 10 chromosomes were counted using classical staining and light microscopy, but 12 chromosomes were resolved by electrophoretic karyotype analysis (Xu et al. 1995) and confirmed by molecular mapping (Xu et al. 1996). The lower chromosome number counts based on traditional cytological techniques are likely due to the small size of fungal chromosomes, which are often difficult to resolve with light microscopy. Recently, Tsuchiya and Taga (2001) studied cytological karyotypes of three Cochliobolus spp. (C. heterostrophus, C. carbonum, and $C$. sativus) using the germ tube burst method (GTBM) and found 15 or 16,13 or 15 , and 15 chromosomes for the three respective species. Their estimations are in agreement with previous reports by Tzeng and associates (1992) for C. heterostrophus (15 or 16 chromosomes), Ahn and Walton (1996) for C. carbonum (13 chromosomes), and the results for $C$. sativus (15 chromosomes) in this study. Therefore, a combination of different methods, including electrophoretic karyotype analysis, telomere probe hybridization, and GTBM, may be the best means to obtain an accurate karyotype of fungi.

The results of this study suggest that at least five chromosomes of isolate ND90Pr were involved in translocations, using the chromosomes of isolate ND93-1 as a reference. These chromosome structural rearrangements may be the cause of the high chromosome length polymorphisms observed between the two isolates. The low fertility in the cross between ND90Pr and ND93-1 found by ValjavecGratian and Steffenson (1997) may be due, to some extent, to these chromosome translocations. Chromosome translocations appear to be fairly common in fungi and have been reported in several Cochliobolus spp., including $C$. heterostrophus (Tzeng et al. 1992) and C. carbonum (Ahn and Walton 1996).

Several genes of $M$. grisea conferring specificity to different grass species and rice cultivars have been cloned with a mapbased cloning method (Farman and Leong 1998; Kang et al.

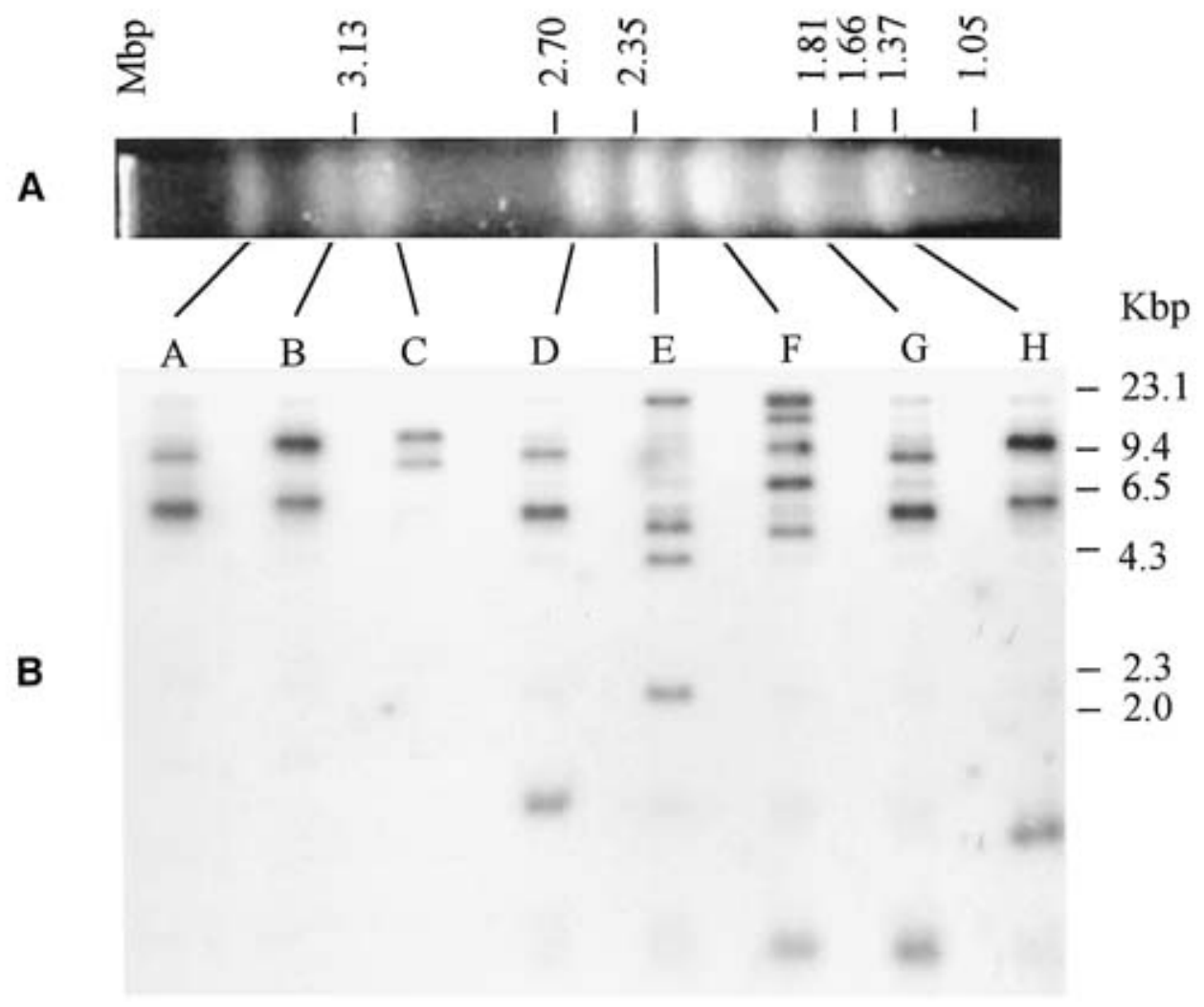

Fig. 3. Resolution of chromosomes from individual contour-clamped homogeneous electric field (CHEF)-separated bands with the telomere probe pNla-17. A, Electrophoretically separated chromosomal DNAs of Cochliobolus sativus isolate ND93-1 from the same gel as shown in Figure 2. B, Hybridization of the telomere probe pNla-17 to BamHI-digested DNA from each CHEF-separated chromosome band (designated as A to $\mathrm{H}$ ). Chromosome bands A, B, and C contained one chromosome because each showed two telomere hybridization bands; chromosome bands D, G, and H contained two chromosomes because each showed two telomere bands plus one high-intensity band which may suggest a doublet; chromosome band E contained two chromosomes because it showed four telomere hybridization bands; and chromosome band F contained four chromosomes because it showed six telomere bands, two of which had higher intensity suggesting a doublet. 
1995; Sweigard et al. 1995; Valent 1997). With the construction of a genetic map in C. sativus, the same strategy can be used to clone genes conferring high virulence on barley and wheat. We recently cloned and characterized several AFLP markers that cosegregated with the barley-specific virulence locus VHvl (S. Zhong and B. J. Steffenson, unpublished data). These initial studies have provided a framework for our ultimate goal of cloning this virulence locus. The same strategy will be used to clone the wheat virulence locus VTa1. Isolation of the two virulence genes will greatly increase our understanding of the molecular interactions of C. sativus with its primary cereal hosts of barley and wheat.

\section{MATERIALS AND METHODS}

\section{Fungal isolates and virulence phenotyping.}

Progeny isolates (104) derived from a cross between the $C$. sativus isolates ND90Pr and ND93-1 (Valjavec-Gratian and Steffenson 1997) were used in this study. The virulence phenotypes of the parental $C$. sativus isolates and their progeny were previously evaluated on the spot blotch differential barley genotypes of 'Bowman', 'ND 5883', and 'ND B112' (Valjavec-Gratian and Steffenson 1997). In a preliminary experiment, parental isolates ND90Pr and ND93-1 ND90Pr also exhibited a differential virulence phenotype on wheat line PI 254042 (Zhong and Steffenson 1999). Thus, the progeny were evaluated for their virulence phenotype on this wheat line. The sowing and growing of the host lines, inoculum preparation, and inoculation protocols were as previously described (Valjavec-Gratian and Steffenson 1997). Infection responses of wheat were scored based on the 0-to-5 rating scale of Adlakha and associates (1984) with ratings of 0 to 3 considered as indicative of low virulence and ratings of 4 to 5 indicative of high virulence.

\section{DNA isolation.}

Genomic DNA was isolated from $C$. sativus using a procedure modified from Yoder (1988) as described previously (Zhong and Steffenson 2001a).

\section{AFLP analysis.}

AFLP analysis was conducted using a method modified from Vos and associates (1995) and described by Zhong and Steffenson (2000). Twenty primer pairs with 2-base extensions were used. Only clear and unambiguous bands were scored. The AFLP markers were named based on the primer combination used and the molecular size in base pairs. For example, EAG/M-CA-207 represents an AFLP marker amplified by primer pair E-AG and M-CA with a molecular size of $207 \mathrm{bp}$.

\section{Cloning of AFLP markers.}

AFLP bands of interest were excised from silver-stained gels and reamplified by PCR using the same primer pairs that generated the original AFLP bands. PCR reactions were performed in a $50-\mu$ l solution containing $1 \times$ buffer $(10 \mathrm{~m}$ M Tris$\mathrm{HCl}$ at $\mathrm{pH} 8.4$ and $50 \mathrm{mM} \mathrm{KCl}), 1.5 \mathrm{mM} \mathrm{MgCl}_{2}, 200 \mu \mathrm{m}$ of dNTP, 1.0 unit of Taq DNA polymerase, $36 \mathrm{ng}$ of each primer, and one excised AFLP band. The PCR profile was denaturation at $95^{\circ} \mathrm{C}$ for $2 \mathrm{~min}$, followed by 30 cycles of $94^{\circ} \mathrm{C}$ for 1 min, $55^{\circ} \mathrm{C}$ for $30 \mathrm{~s}$, and $72^{\circ} \mathrm{C}$ for $1 \mathrm{~min}$. Reamplified PCR products were verified by comparing with the respective AFLP bands in the denaturing polyacrylamide gels and then cloned into the plasmid vector pCR2.1 using the TA cloning kit (Invitrogen, Carlsbad, CA, U.S.A.) according to the supplier's instructions.

\section{Southern hybridization and RFLP analysis.}

DNA clones previously used to construct a genetic map of C. heterostrophus (Tzeng et al. 1992) were used as probes

Table 2. Summary of data on the resolution of chromosome-sized bands separated by CHEF bands combined with telomere probe analysis and hybridization of AFLP and RFLP probes on CHEF blots in isolates ND93-1 and ND90Pr of Cochliobolus sativus ${ }^{\mathrm{a}}$

\begin{tabular}{|c|c|c|c|}
\hline Isolate, CHEF band ${ }^{\mathrm{b}}$ & $\begin{array}{l}\text { Telomere } \\
\text { fragments }^{\mathrm{c}}\end{array}$ & $\begin{array}{l}\text { Number of } \\
\text { chromosomes }^{d}\end{array}$ & AFLP and RFLP probes hybridized \\
\hline \multicolumn{4}{|l|}{ ND93-1 } \\
\hline A & 2 & 1 & B264, C50, G131, E-AT/M-AC-584, E-AC/M-CG-195, E-AG/M-CC-753 \\
\hline B & 2 & 1 & $\mathrm{~B} 84, \mathrm{~B} 107, \mathrm{~B} 312$ \\
\hline $\mathrm{C}$ & 2 & 1 & В277, В283, В421, В429, C9, G38а, B125a, G311, B91, E-GC/M-AG-237, E-GG/M-AC-813 \\
\hline $\mathrm{D}$ & 4 & 2 & B71, B154, B301, B403, B405, C246, G127, G214, E-AG/M-CA-200, E-AG/M-CC-148 \\
\hline E & 4 & 2 & B125b, B246, B285, G29, HMGPCR1, E-AC/M-CC-275a \\
\hline $\mathrm{F}$ & 8 & 4 & $\begin{array}{l}\text { B43, B114, B329, C72, G38b, G300, CSMAT, E-AG/M-CC-400, E-AG/M-CC-402, } \\
\text { E-AC/M-AT-882, E-AC/M-CG-335, E-AT/M-CC-499, E-AT/M-AC-271, E-GC/M-AG-633, } \\
\text { E-GG/M-AC-286 }\end{array}$ \\
\hline G & 4 & 2 & B416, C193, G199, E-AT/M-CC-182, E-GC/M-AG-170, E-AT/M-CC-182 \\
\hline $\mathrm{H}$ & 4 & 2 & B257, C3, G235, E-AC/M-CC-275b \\
\hline \multicolumn{4}{|l|}{ ND90Pr } \\
\hline A & 2 & 1 & B84, B285, B107, HMGPCR1, E-AC/M-CC-275a \\
\hline B & 2 & 1 & B264, C50, G131, E-AT/M-AC-584, E-AC/M-CG-195, E-AG/M-CC-753 \\
\hline $\mathrm{C}$ & 2 & 1 & B277, B283, B403, B405, B421, B429, C9, G38a, G127, G214, G311, E-GC/M-AG-237 \\
\hline $\mathrm{D}$ & 4 & 2 & $\begin{array}{l}\text { B125a, B154, C246, B301, CSMAT, E-AG/M-CC-400, E-AG/M-CC-402, E-AG/M-CA-200, } \\
\text { E-GC/M-AG-104 }\end{array}$ \\
\hline E & 2 & 1 & B91, B416, C193, E-GC/M-AG-170 \\
\hline F & 2 & 1 & G38b, GG/AC-813, GC/AG-237, E-AT/M-AC-271 \\
\hline G & 8 & 4 & $\begin{array}{l}\text { B246, G29, B43, B114, B329, C72, G300, G199, E-AC/M-AT-882, E-AC/M-CG-335, } \\
\text { E-AT/M-CC-499, E-GC/M-AG-633, E-GG/M-AC-286 }\end{array}$ \\
\hline $\mathrm{H}$ & 2 & 1 & B71 \\
\hline I & 2 & 1 & B257, E-AC/M-CC-275b \\
\hline $\mathrm{J}$ & 2 & 1 & B125b \\
\hline $\mathrm{K}$ & 2 & 1 & $\mathrm{C} 3, \mathrm{G} 235$ \\
\hline
\end{tabular}

${ }^{\mathrm{a}} \mathrm{CHEF}=$ contour-clamped homogeneous electric field; AFLP = amplified fragment length polymorphism; and RFLP = restriction fragment length polymorphism.

${ }^{\mathrm{b}}$ CHEF bands are chromosome-sized bands resolved under conditions for resolving lower (1.0 to $\left.2.5 \mathrm{Mbp}\right)$ and higher (2.5 to $\left.4.0 \mathrm{Mbp}\right)$ molecular weight bands, respectively.

${ }^{\mathrm{c}}$ Number of telomere fragments was based on the telomere hybridization bands on blots with restriction enzyme-digested DNA from individual CHEF bands.

${ }^{\mathrm{d}}$ Number of chromosome was determined based on the number of telomere fragments resolved with both BamHI and HindIII. 
for the RFLP analysis of $C$. sativus. These clones were generous gifts from C. R. Bronson (Iowa State University, Ames, IA, U.S.A.). The mating type genes cloned from $C$. sativus (Zhong and Steffenson 2001a) also were used as probes to map the mating type locus. Southern hybridization was performed as previously described (Zhong and Steffenson 2001a).

\section{Data analysis and map construction.}

The AFLP and RFLP markers detected between parental isolates ND90Pr and ND93-1 were evaluated on the 104 progeny isolates and tested for deviations from an expected 1:1 segregation ratio using the $\chi^{2}$ test $(\alpha=0.05)$. Linkage analysis was performed using the computer program MAPMAKER version 2 .0 (Lander et al. 1987). A minimum LOD value of 4.0 and a maximum theta of 0.3 were used to establish linkage relationships between markers. The Kosambi mapping function (Kosambi 1944) was used to convert recombination frequency to map distance in centimorgans.
Electrophoretic karyotyping.

Preparation of protoplasts. Protoplasts of $C$. sativus were prepared using a procedure adapted from Ciuffetti and associates (1997). Briefly, conidia of the fungal isolates were harvested from 8- to 10-day-old cultures and inoculated into a $250-\mathrm{ml}$ glass flask containing 50 to $75 \mathrm{ml}$ of $1 \times$ potato dextrose broth for a final concentration of $10,000 \mathrm{conidia} / \mathrm{ml}$. The flask was placed on a rotary shaker $(150 \mathrm{rpm})$ and incubated overnight at room temperature $\left(22\right.$ to $\left.25^{\circ} \mathrm{C}\right)$. Mycelium was separated from the culture solution by filtration through four layers of cheesecloth. Harvested mycelium was washed first with sterile distilled water and then osmotic magnesium (OM) buffer (1.2 $\mathrm{M} \mathrm{MgSO}_{4}$ and $10 \mathrm{mM} \mathrm{Na}$ phosphate at $\mathrm{pH} 5.8$ ) (Yelton et al. 1984). The washed mycelium was suspended in $4 \mathrm{ml}$ of $\mathrm{OM}$ buffer in a $50-\mathrm{ml}$ flask before $4 \mathrm{ml}$ of filter-sterilized enzyme solution (10 to $20 \mathrm{mg}$ of Novozyme [Interspex Products Inc., Foster City, CA, U.S.A.], $10 \mathrm{mg}$ of Driselase [Sigma-Aldrich, St. Louis], and $0.5 \mathrm{ml}$ of trapping buffer [0.6

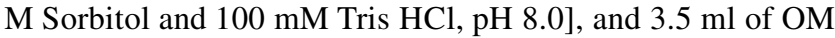

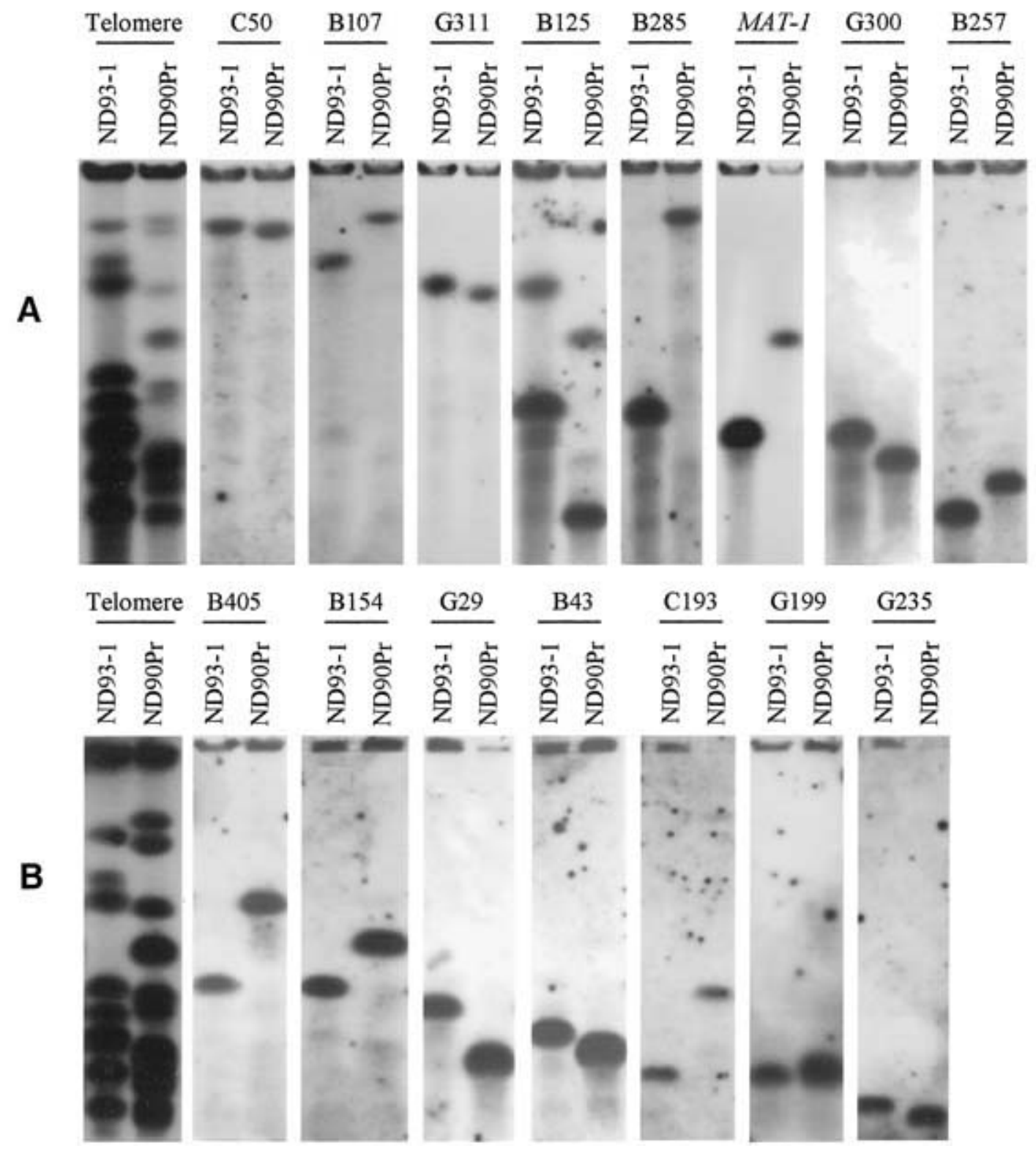

Fig. 4. Hybridization of DNA probes to contour-clamped homogeneous electric field (CHEF)-separated chromosomes of Cochliobolus sativus isolates ND93-1 and ND90Pr. A, and B, represent CHEF blots from two independent runs under CHEF conditions for resolving higher molecular weight chromosome bands (2.5 to $4.0 \mathrm{Mbp}$ ). The same CHEF blots were stripped before reuse for a different probe. Hybridization of representative markers (probes) to the CHEF blots is shown. 
buffer) was added. After incubation on a rotary shaker (50 $\mathrm{rpm})$ at $27^{\circ} \mathrm{C}$ for 2 to $3 \mathrm{~h}$, the mycelium suspension was filtered through a Nitex filter $(75 \mu \mathrm{M})$ (Tetko Inc., Depew, NY, U.S.A.), and the resulting solution containing protoplasts was centrifuged at $2,000 \times g$ for 8 to $10 \mathrm{~min}$. The pellet was resus- pended in sorbitol EDTA (SE) buffer (1.2 M sorbitol and 50 mM EDTA), and the concentration was adjusted to $2 \times 10^{8}$ protoplasts/ml with SE. To make gel plugs, the protoplast solution was mixed with an equal volume of $1.6 \%$ low-melting agarose in $\mathrm{SE}$ at $50^{\circ} \mathrm{C}$. This mixture was pipetted into plug

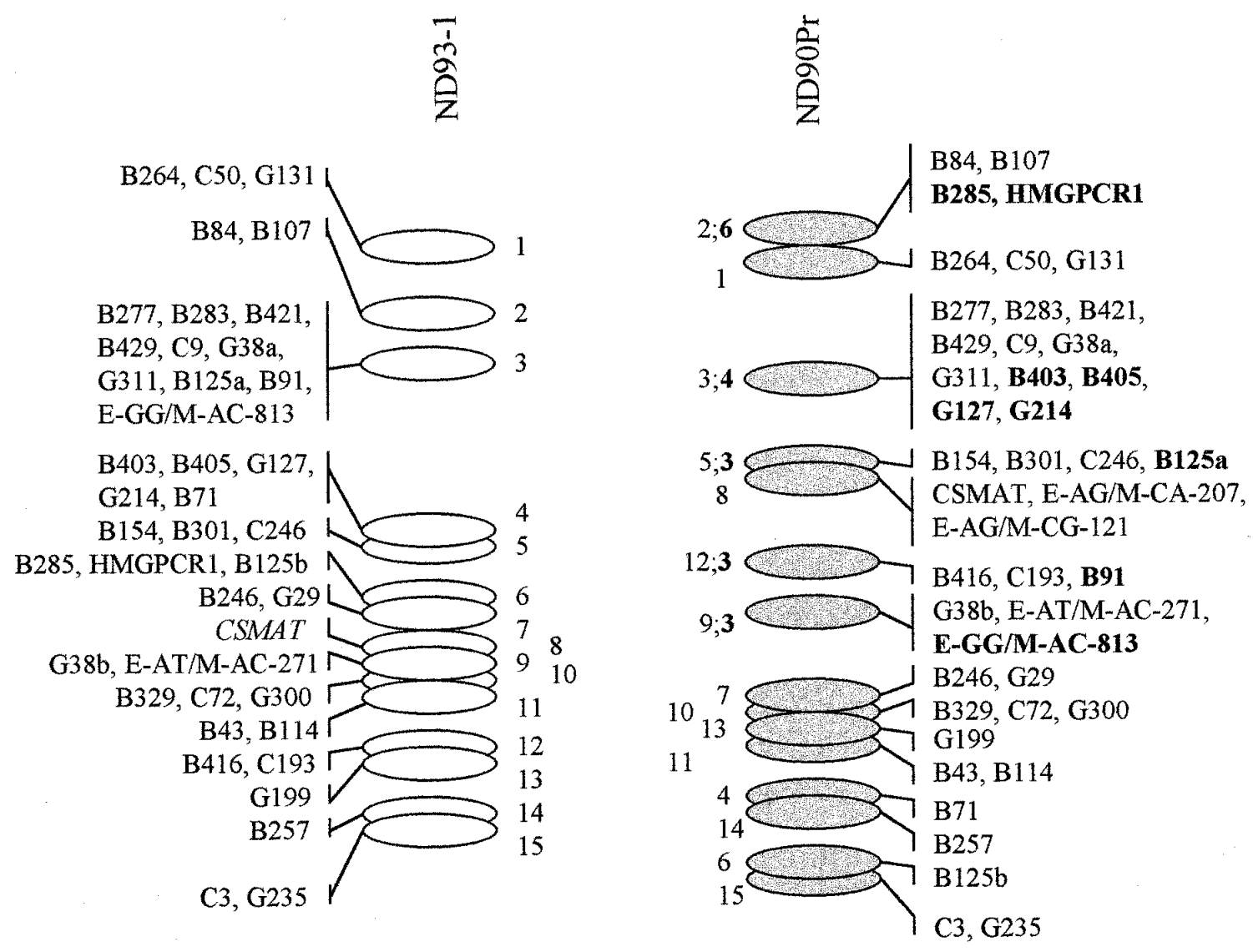

Fig. 5. Diagram of electrophoretic karyotypes of the Cochliobolus sativus parental isolates ND93-1 and ND90Pr based on contour-clamped homogeneous electric field (CHEF) analyses and hybridization patterns with DNA probes. The karyotype of isolate ND93-1 was chosen as the reference for numbering the chromosomes with the largest designated chromosome 1. Representative restriction fragment length polymorphism and amplified fragment length polymorphism markers associated with their respective chromosomes are indicated on left side for ND93-1 and right side for ND90Pr. Five chromosomes $(2 ; 6,3 ; 4,5 ; 3,12 ; 3$, and 9;3) of isolate ND90Pr contain DNA markers (bold) from different chromosomes in isolate ND93-1, suggesting that they were derived from chromosome translocations.

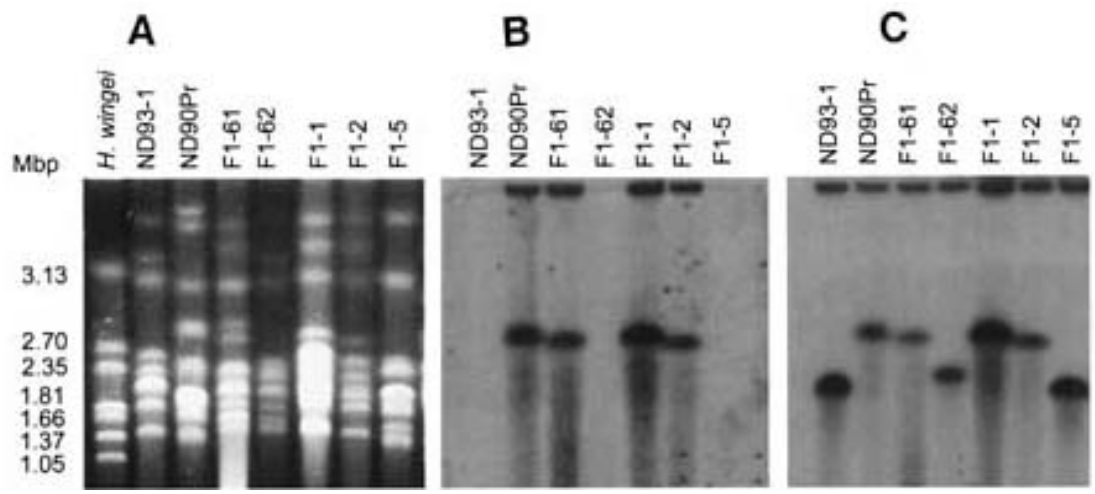

Fig. 6. Electrophoretic karyotype and Southern hybridization analyses of the Cochliobolus sativus parental isolates ND93-1 and ND90Pr and five of their progeny. A, Chromosome bands separated by contour-clamped homogeneous electric field (CHEF) under the same conditions as described in Figure 2. B, Autoradiography of the CHEF blot probed with the cloned amplified fragment length polymorphism marker E-AG/M-CA-207. E-AG/M-CA-207 only hybridized with a 2.80-Mbp chromosome of isolate ND90Pr and a chromosome of three progeny $\left(\mathrm{F}_{1}-1, \mathrm{~F}_{1}-2\right.$, and $\left.\mathrm{F}_{1}-61\right)$ exhibiting high virulence on barley cv. Bowman. E-AG/M-CA-207 is absent from the ND93-1 genome. C, The same CHEF blot probed with the mating type gene CsMAT-1 (Zhong and Steffenson 2001a) after stripping. CsMAT-1 was localized on the same chromosome as E-AG/M-CA-207 in isolate ND90Pr and hybridized with a 2.2-Mbp chromosome of isolate ND93-1 and a chromosome in all six $\mathrm{F}_{1}$ progeny. 
molds and solidified in a refrigerator at $4{ }^{\circ} \mathrm{C}$ for $10 \mathrm{~min}$. Agarose plugs were placed in NDS buffer (0.5 M EDTA, $10 \mathrm{mM}$ Tris at $\mathrm{pH} 8.0,1 \%$ Sarkosyl, and Proteinase $\mathrm{K}$ at 0.5 to 1 $\mathrm{mg} / \mathrm{ml}$ ), incubated overnight at 22 to $25^{\circ} \mathrm{C}$, washed twice with $0.5 \mathrm{M}$ EDTA, and stored in $50 \mathrm{mM}$ EDTA at $4^{\circ} \mathrm{C}$ until needed.

Separation of chromosome-sized DNAs. Agarose plugs containing the fungal protoplasts were loaded in a $0.8 \%$ chromosome-grade agarose gel, and then chromosome-sized DNAs were separated in $1 \times$ Tris-acetate-EDTA buffer at $14^{\circ} \mathrm{C}$ with a CHEF system (CHEF DRII; Bio-Rad, Richmond, CA, U.S.A.). Two different running conditions were used. To resolve lower molecular weight chromosome bands (1.0 to 2.5 Mbp), we adapted the conditions used by Russell and Mills (1993). The voltage, switch time, and running time were 3.0 $\mathrm{V} / \mathrm{cm}, 480$ to $600 \mathrm{~s}$, and $96 \mathrm{~h}$, respectively. To resolve higher molecular weight chromosome bands (2.5 to $4.0 \mathrm{Mbp}$ ), a voltage and switch time of $2.0 \mathrm{~V} / \mathrm{cm}$ and 1,200 to $960 \mathrm{~s}$ for $24 \mathrm{~h}$, followed by $2.5 \mathrm{~V} / \mathrm{cm}$ and 960 to $480 \mathrm{~s}$ for $96 \mathrm{~h}$ were used. Separated chromosomes were stained with ethidium bromide or SYBR gold nucleic acid gel stain (Molecular Probes, Eugene, OR, U.S.A.). After photography, the separated chromosome-sized DNAs were blotted onto a Hybond $\mathrm{N}+$ filter as described by $\mathrm{Xu}$ and associates (1995). Chromosome sizes of C. sativus were estimated by comparison with commercially prepared chromosomes of $H$. wingei and S. pombe (Bio-Rad).

Resolution of chromosomes with telomere probe. Some chromosomes could not be resolved with CHEF alone, because they comigrated in the gel because of their similar size. The clone pNla-17, containing a telomere repeat sequence [TTAGGG] $]_{18}$ from Fusarium oxysporum (Powell and Kistler 1990), was used as probe to determine the number of chromosomes from a single CHEF band. The rationale for this approach was that each chromosome contains two telomeres that are detectable with the telomere probe (Mills et al. 1995). Chromosome DNAs were separated with CHEF as described above, except that a $0.8 \%$ low-melting agarose gel was used. Individual chromosome bands were excised and DNA was isolated with Gelase (Epicentre, Madison, WI, U.S.A.) according to the supplier's instructions. DNA was digested with BamHI or HindIII, separated in a $0.9 \%$ agarose gel, and probed with the telomere sequence using the methods described for the RFLP analysis.

\section{Assignment of linkage groups and DNA markers to CHEF-separated chromosomes.}

To assign the genetic linkage groups constructed with molecular markers to specific CHEF-separated chromosomes, at least one of the AFLP or RFLP markers mapped in each linkage group was used as a probe to hybridize with DNA blots made from the CHEF-separated chromosomes. Probe labeling, hybridization, and filter washing were done using the protocols as described for RFLP analysis.

\section{ACKNOWLEDGMENTS}

We thank C. R. Bronson and H. C. Kistler for providing the C. heterostrophus RFLP clones and the telomere clone, respectively. This study was supported in part by the American Malting Barley Association.

\section{LITERATURE CITED}

Adlakha, K. L., Wilcoxson, R. D., and Raychaudhuri, S. P. 1984. Resistance of wheat to leaf spot caused by Bipolaris sorokiniana. Plant Dis. 68:320-321.

Ahn, J.-H., and Walton, J. D. 1996. Chromosomal organization of TOX2, a complex locus controlling host-selective toxin biosysnthesis in Cochliobolus carbonum. Plant Cell 8:887-897.

Ahn, S., and Tanksley, S. D. 1993. Comparative linkage group maps of the rice and maize genomes. Proc. Natl. Acad. Sci. U.S.A. 90:7980-7984.
Arie, T., Christiansen, S. K., Yoder, O. C., and Turgeon, B. G. 1997. Efficient cloning of ascomycete mating type genes by PCR amplification of the conserved MAT HMG box. Fungal Genet. Biol. 21:118-130.

Bennetzen, J. L., and Freeling, M. 1993. Grasses as a single genetic system: Genome composition, colinearity and compatibility. Trends Genet. 9:259-261.

Botstein, D., White, R. L., Skolnick, M., and Davis, R. W. 1980. Construction of a genetic linkage group in man using restriction fragment length polymorphisms. Am. J. Hum. Genet. 32:314-331.

Ciuffetti, L. M., Tuori, R. P., and Gaventa, J. M. 1997. A single gene encodes a selective toxin causal to the development of tan spot of wheat. Plant Cell 9:135-144.

Devos, K. M., Wang, Z. M., Beales, J., Sasaki, T., and Gale, M. D. 1998. Comparative genetic maps of foxtail millet (Setaria italica) and rice (Oryza sativa). Theor. Appl. Genet. 96:63-68.

Farman, M. L., and Leong, S. A. 1998. Chromosome walking toward Avr1-CO39 avirulence gene of Magnaporthe grisea: Discrepancy between the physical and genetic maps. Genetics 150:1049-1058.

Fincham, J. R. S. 1983. Genetics. 3rd ed. Jones and Bartlett Publishers, Boston.

Guzman, D., Garber, R. C., and Yoder, O. C. 1982. Cytology of meiosis and chromosome number of Cochliobolus heterostrophus (Ascomycetes). Can. J. Bot. 60:1138-1141.

Hosford, R. M., Jr., Solangi, G. R. M., and Kiesling, R. L. 1975. Inheritance in Cochliobolus sativus. Phytopathology 65:699-703.

Hrushovetz, S. B. 1956. Cytological studies of ascus development in Cochliobolus sativus. Can. J. Bot. 34:641-651.

Huang, H. C., and Tinline, R. D. 1974. Somatic mitosis in haploid and diploid strains of Cochliobolus sativus. Can. J. Bot. 52:1561-1568.

Hulbert, S. H., Ilott, T. W., Legg, E. J., Lincoln, S. E., Lander, E. S., and Michelmore, R. W. 1988. Genetic analysis of the fungus, Bremia lactucae, using restriction fragment length polymorphisms. Genetics 120:947-958.

Kang, S., Sweigard, J. A., and Valent, B. 1995. The $P W L$ host specificity gene family in the blast fungus Magnaporthe grisea. Mol. PlantMicrobe Interact. 8:939-948.

Kline, D. M., and Nelson, R. R. 1971. The inheritance factors in Cochliobolus sativus conditioning lesion induction on gramineous hosts. Phytopathology 61:1052-1054.

Kosambi, D. D. 1944. The estimation of map distance from recombination values. Ann. Eugen. 12:172-175.

Lander, E. S., Green, P., Abrahamson, J., Barlow, A., Daly, M. J., Lincoln, S. E., and Newburg, L. 1987. MAPMAKER: An interactive computer package for constructing primary genetic linkage maps of experimental and natural populations. Genomics 1:174-181.

Mathre, D. E. 1997. Compendium of Barley Diseases. 2nd ed. American Phytopathological Society Press, St. Paul, MN, U.S.A.

Mills, D., McCluskey, K., Russell, B. W., and Agnan, J. 1995. Electrophoretic karyotyping: Method and Applications. Pages 81-96 in: Molecular Methods in Plant Pathology. U. S. Singh and R. P. Singh, eds. SpringerVerlag, Berlin.

Orbach, M., Vollrath, D., Davis, R. W., and Yanofsky, C. 1988. An electrophoretic karyotype of Neurospora crassa. Mol. Cell Biol. 8:1469-1473.

Orbach, M. J., Farrall, L., Sweigard, J. A., Chumley, F. G., and Valent, B. 2000. A telomeric avirulence gene determines efficacy for the rice blast resistance gene Pi-ta. Plant Cell 12:2019-2032.

Pongam, P., Osborn, T. C., and Williams, P. H. 1999. Genetic analysis and identification of amplified fragment length polymorphism markers linked to the alm1 avirulence gene of Leptosphaeria maculans. Phytopathology 88:1068-1072.

Powell, W. A., and Kistler, H. C. 1990. In vivo rearrangement of foreign DNA by Fusarium oxysporum produces linear self-replicating plasmids. J. Bacteriol. 172:3163-3171.

Russell, B. W., and Mills, D. 1993. Electrophoretic karyotypes of Tilletia caries, $T$. controversa, and their $\mathrm{F}_{1}$ progeny: Further evidence for conspecific status. Mol. Plant-Microbe Interact. 6:66-74.

Skinner, D. Z., Budde, A. D., Farman, M. L., Smith, J. R., Leung, H., and Leong, S. A. 1993. Genome organization of Magnaporthe grisea: Genetic map, electrophoretic karyotype, and occurrence of repeated DNAs. Theor. Appl. Genet. 87:545-557.

Sweigard, J. A., Carroll, A. M., Kang, S., Farrall, L., Chumley, F. G., and Valent, B. 1995. Identification, cloning, and characterization of PWL2, a gene for host species specificity in the rice blast fungus. Plant Cell 7:1221-1233.

Tanksley, S. D., Ganal, M. W., Prince, J. P., de Vicente, M. C., Bonierbale, M. W., Broun, P., Fulton, T. M., Giovannoni, J. J., Grandillo, S., Martin, G. B., Messeguer, R., Miller, J. C., Miller, L., Patterson, A. H., Pineda, O., Roder, M. S., Wing, R. A., Wu, W., and Young, N. D. 1992. High density molecular linkage maps of tomato and potato genomes. Genetics 132:1141-1160. 
Tsuchiya, D., and Taga, M. 2001. Cytological karyotyping of three Cochliobolus spp. by the germ tube burst method. Phytopathology 91:354-360.

Turgeon, B. G., Bohlmann, H., Ciuffetti, L. M., Christiansen, K. S., Yang, G., Schafer, W., and Yoder, O. C. 1993. Cloning and analysis of the mating type genes from Cochliobolus heterostrophus. Mol. Gen. Genet. 238:270-284.

Tzeng, T. H., Lyngholm, L. K., Ford, C. F., and Bronson, C. R. 1992. A restriction fragment length polymorphism map and electrophoretic karyotype of the fungal maize pathogen Cochliobolus heterostrophus. Genetics 130:81-96.

Valent, B., 1997. The rice blast fungus Magnaporthe grisea. Pages 37-54 in: The Mycota V: Plant Relationships Part B. G. C. Carroll and P. Tudzynski, eds. Springer-Verlag, Berlin.

Valjavec-Gratian, M., and Steffenson, B. J. 1997. Genetics of virulence in Cochliobolus sativus and resistance in barley. Phytopathology 87:11401143.

van der Lee, T., De Witte, I., Drenth, A., Alfonso, C., and Govers, F. 1997. AFLP linkage map of the Oomycete Phytophthora infestans. Fungal Genet. Biol. 21:278-291.

Vos, P., Hogers, R., Bleeker, M., Reijans, M., van de Lee, T., Hornes, M., Freijters, A., Pot, J., Peleman, J., Kuiper, M., and Zabeau, M. 1995. AFLP: A new concept for DNA fingerprinting. Nucleic Acids Res. 23:4407-4414.

Whisson, S. C., Drenth, A., Maclean, D. J., and Irwin, J. A. G. 1995. Phytophthora sojae avirulence genes, RAPD, and RFLP markers used to construct a detailed genetic linkage map. Mol. Plant-Microbe Interact. 8:988-995.

Wiese, M. V. 1977. Compendium of Wheat Diseases. American Phytopa- thological Society Press, St. Paul, MN, U.S.A

Williams, J. G. K., Kubelik, A. R., Livak, K. J., Rafalski, J. A., and Tingey, S. V. 1991. DNA polymorphisms amplified by arbitrary primers are useful as genetic markers. Nucleic Acids Res. 18:6531-6535.

Xu, J. R., and Leslie, J. F. 1996. A genetic map of Gibberella fujikuroi mating population A (Fusarium moniliforme). Genetics 143:175-189.

Xu, J.-R., Yan, K., Dickman, M. B., and Leslie, J. F. 1995. Electrophoretic karyotypes distinguish the biological species of Gibberella fujikuroi (Fusarium Section Liseola). Mol. Plant-Microbe Interact. 8:74-84.

Yelton, M. M., Hamer, J. E., and Timberlake, W.E. 1984. Transformation of Aspergillus nidulans by using a trpC plasmid. Proc. Natl. Acad. Sci. U.S.A. 81:1470-1474.

Yoder, O. C. 1988. Cochliobolus heterostrophus, cause of southern corn leaf blight. Pages 93-112 in: Advances in Plant Pathology Vol. 6. D. S Ingram and P. H. Williams, eds. Academic Press, London.

Young, N. D. 1990. Potential applications of map-based cloning to plant pathology. Physiol. Mol. Plant Pathol. 37:81-94.

Zhong, S., and Steffenson, B. J. 1999. Molecular mapping of genes conferring virulence on barley and wheat in Cochliobolus sativus. Page 122 in: 20th Fungal Genet. Conf. Pacific Grove, CA, U.S.A.

Zhong, S., and Steffenson, B. J. 2000. A simple and sensitive silver-staining method for detecting AFLP markers in fungi. Fungal Genet Newsl. 47:101-102.

Zhong, S., and Steffenson, B. J. 2001a. Genetic and molecular characterization of mating type genes in Cochliobolus sativus. Mycologia 93:852-863.

Zhong, S., and Steffenson, B. J. 2001b. Virulence and molecular diversity in Cochliobolus sativus. Phytopathology 91:469-476. 